\title{
Improved Node Matrix Optimization Method of Test Points at Circuit Board
}

\author{
LI Feng-yu ${ }^{1}$, YAN Yu-kun ${ }^{1}$, ZHANG Long-fei ${ }^{1}$, CHEN Zhi ${ }^{2}$, LIAO Long-tao ${ }^{2}$, \\ $\mathrm{XIAO} \mathrm{\textrm {Kai } ^ { 2 }}$
}

1125 mailbox, JIE FANG road No.717, Wuhan 430033 China

\begin{abstract}
${ }^{2}$ Science and technology on reactor system design technology laboratory, Nuclear Power Institute of China, Chengdu 610041, China
\end{abstract}

Keywords: circuit board, fault diagnosis, detection, optimization method

\begin{abstract}
Graph theory is applied in this paper to optimization design of test point set in circuit board fault diagnosis. The optimal detection node set of a circuit board can be established by a improved node matrix. The improved node matrix can also regenerate topology network graph of a circuit, which reflects the relationship among every node and the working status of all components in the circuit.
\end{abstract}

\section{Introduction}

For restriction of limited nodes which can be reached, the fault information for diagnosis is not sufficient and the fault position can not be determined precisely. Selection of test points is directly related to how much fault information can be obtained, diagnosis precision and efficiency. The optimal fault detection point defined in reference [1] includes most information of fault source. reference [1] also considers that test point set should cover fault source information as far as possible. Principle of optimized method for test point is expanded in this paper.

\section{Topology structure model of circuit}

Topology structure of a network can be described by a graph, or a matrix. Let a electrical network composed of $l$ branching-offs and $n$ nodes, as shown in figure 1 . All branching-offs and nodes are numbered arbitrarily. Therefore the association properties between branching-offs and nodes can be expressed by a $n \times b$ order matrix $M_{c}$. A row of $M_{c}$ is corresponding for a node, and a column of $M_{c}$ for a branching-off. Element $m_{j k}$ of matrix $M_{c}$ is defined as follows:

(1) $m_{j k}=1$, if $k$-th branching-off is dependent on $j$-th node, and $k$-th branching-off departs from $j$-th node;

(2) $m_{j k}=-1$, if $k$-th branching-off is dependent on $j$-th node, and $k$-th branching-off directs towards $j$-th node;

(3) $m_{j k}=0$, if $k$-th branching-off is independent on $j$-th node.

$M_{c}$ is called association matrix of a electrical network between nodes and branching-offs. Association matrix of a electrical network figure shown in figure 1 is formula (1).

$$
M_{c}=n \begin{array}{rccccccc}
b_{1} & b_{2} & b_{3} & b_{4} & b_{5} & b_{6} & b_{7} \\
& n 1 \\
& n 4
\end{array}\left(\begin{array}{ccccccc}
1 & 1 & 0 & 1 & 0 & 0 & 1 \\
0 & -1 & 0 & 0 & 1 & 1 & -1 \\
-1 & 0 & 1 & 0 & 0 & -1 & 0 \\
0 & 0 & -1 & -1 & -1 & 0 & 0
\end{array}\right)
$$




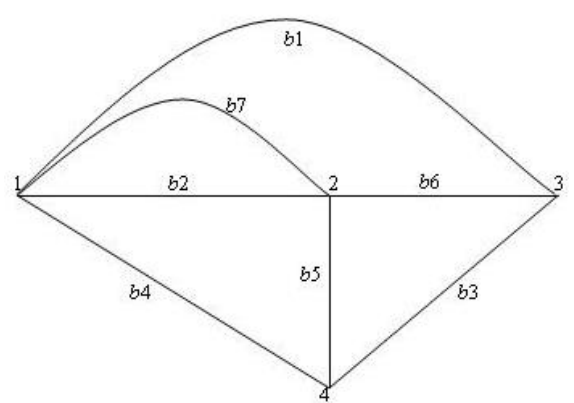

Fig. 1 Structure of a simple circuit network

It can be found that every column of a association matrix has only two non-zero elements $(+1)$ and $(-1)$, and $\sum_{j=1}^{n} M_{j}=[0 \cdot 0 \cdot 0 \cdots]_{1 \times l}$. Therefore association matrix is linear correlation. Giving a $(n-1) \times l$ order association matrix can determine a directed graph which is composed of $l$ branching-offs and $n$ nodes.

\section{Improved node matrix model}

Rows and columns of adjacent node matrix $\mathrm{G}$ defined in reference [1] rank identically. If two nodes are connected, the corresponding element of matrix is 1. Otherwise, the corresponding element is 0 . Topology structure of an electrical network can be described by this way, but whether branch has element or not can not be determined. The improved node matrix is shown as follow.

$$
\begin{aligned}
& \begin{array}{llll}
n_{1} & n_{2} & n_{3} & n_{4}
\end{array} \\
& G=\underset{n_{2}}{n_{3}} n_{4}\left(\begin{array}{llll}
i & 2 & 1 & 1 \\
2 & i & 1 & 1 \\
1 & 1 & i & 1 \\
1 & 1 & 1 & i
\end{array}\right) \\
& \mathrm{G}_{\triangle \mathrm{I}}=\underset{n_{2}}{n_{1}} n_{3}\left(\begin{array}{cccc}
n_{1} & n_{2} & n_{3} & n_{4} \\
& 2 & 1 & 1 \\
& i & 1 & 1 \\
& & i & 1 \\
& & & i
\end{array}\right)
\end{aligned}
$$

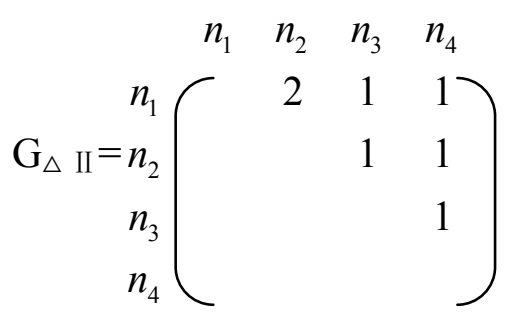

A matrix composed of $n$ nodes has $n^{2}$ elements. Because the matrix is symmetrical, $\frac{n \cdot(n+1)}{2}$ elements are duplicated. All $n$ diagonal elements are $i$. Number of the effective matrix elements is $\frac{n \cdot(n-1)}{2}$. The matrix can be simplified to a triangular matrix.

If a branch is broken, 1 should be subtracted from the element $G_{(a, b)}$ corresponding to two nodes $\left(\mathrm{n}_{\mathrm{a}}, \mathrm{n}_{\mathrm{b}}\right)$ associated with the branch.

If a branch is direct shot, 1 should be subtracted from the element $\mathrm{G}_{(\mathrm{a}, \mathrm{b})}$ corresponding to two 
nodes $\left(\mathrm{n}_{\mathrm{a}}, \mathrm{n}_{\mathrm{b}}\right)$ associated with the branch and add $i$.

Let branching-off $b 5$ broken, $b 3$ and $b 7$ direct short in figure 1, we get

$$
\begin{aligned}
& \mathrm{G}=n_{2} n_{3}\left(\begin{array}{cccc}
n_{1} & n_{2} & n_{3} & n_{4} \\
n_{4} & 1+i & 1 & 1 \\
1+i & i & 1 & 0 \\
1 & 1 & i & i \\
1 & 0 & i & i
\end{array}\right)
\end{aligned}
$$

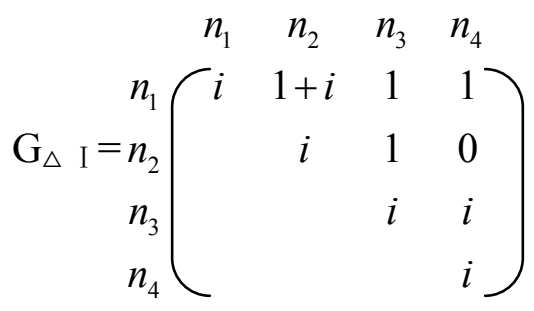

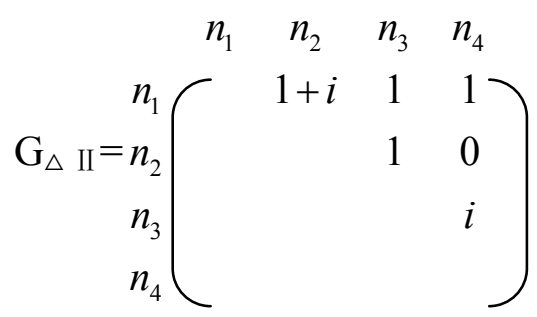

\section{Realization of optimized test point model based on improved node matrix}

3.1 Realization method

Optimal detection node set $V$ can be established from improved node matrix. Let $b 3$ and $b 7$ direct short in figure 1 , and follow the example (7), we get a $(\mathrm{n}-1) \times(\mathrm{n}-1)$ upper triangular matrix

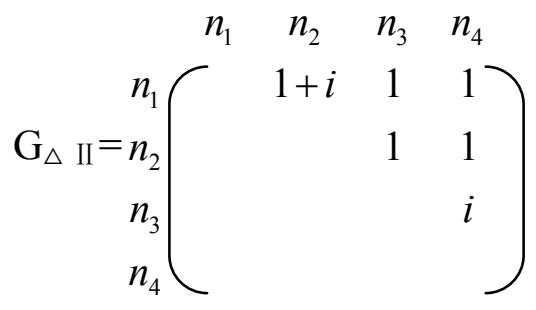

If $b 3$ branch is not associated with test points $\mathrm{n} 1$ and $\mathrm{n} 2$, then

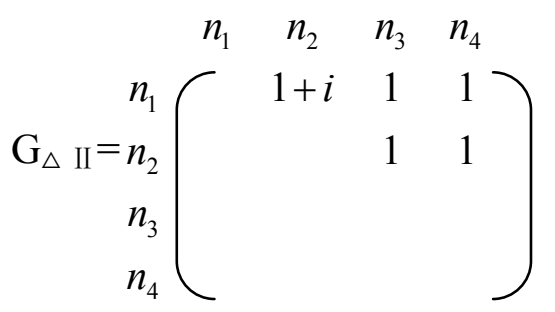

Let the different element between equation (8) and (9) is ' 0 '. The fewer elements ' 0 ', the more node set $V$ covers branches.

The more elements ' 1 ' in equation (9), the less repetition degree of branch associated with nodes in set $V$.

The smaller the variance $\operatorname{Var}\left\{\right.$ num $\left.\left[T\left(n_{a}\right)\right], \operatorname{num}\left[T\left(n_{b}\right)\right], \cdots n u m\left[T\left(n_{t}\right)\right]\right\}$, the more average the number of branching-off associated with a node.

3.2 Essence of the algorithm 
Typical combinational optimization problems are set-covering, enchasement, binning and travelling salesman problem(TSP).

The set-covering problem can be described as following example.

$$
\begin{aligned}
\mathrm{c} & =\left[\begin{array}{llllll}
10 & 15 & 11 & 10 & 8 & 2
\end{array}\right] \\
\mathrm{A} & =\left[\begin{array}{llllll}
1 & 1 & 0 & 1 & 0 & 0 \\
0 & 0 & 1 & 1 & 0 & 0 \\
1 & 1 & 1 & 0 & 0 & 0 \\
0 & 0 & 0 & 0 & 1 & 1 \\
0 & 0 & 1 & 0 & 0 & 1 \\
0 & 0 & 0 & 1 & 1 & 0
\end{array}\right]
\end{aligned}
$$

Its solution is $\mathrm{x}=[1,0,1,0,1,0]$ and cost is 29 . Truck dispatch, resource distribution and location of equipment are also set covering problem.

\section{Conclusion}

Principles of building test set condition each other. A set does not exist meeting all condition. Stressing one principle does not accord with engineering practice. Neglecting branching-off coverage won't detect fault on circuit board. Neglecting repetition degree of branching-off associated with nodes will reduce precision of fault location. Too many branching-off associated with a node will damage status resolution of different branching-off. Few nodes will gather many faults on one node. With the electrical network spreading, too many nodes will make processing difficult and consume much resource. Node number relates to specific demand. You can set more nodes in production line and less node in detection. Optimal test set is realized by constructing adaptation degree function and each restriction condition.

Promoted node matrix can discover abnormality in circuit, reflect faulty status of electrical network, and is convenient for program.

\section{References}

[1]Balinski M. Interger programming: method, uses and computation[J], Management Science,1965, 12(3), p. 253-313.

[2]Garey M. Johnson D. Computers and Intractability: A Guide to the Theory of NP-Completeness[C]. New York: W H Freeman, 1979: p. 50-55.

[3]Valenta J.Capital equipment decisions: a model for optimal systems interfacing[C]. Masszchusetts Institute of Technology: Master's thesis, 1969: p. 30-33.

[4]Walker W. Using the set-covering problem to assign fire companies to fire house[J]. European Journal of Operational Research, 1974, 22: p. 275-277.

[5] LI Feng-yu. Double PI Control for Nuclear Steam Generator Water Level[J]. Atomic Energy Science and Technology, 2010, 44(Suppl.):279-283. 